OPEN ACCESS

Edited by:

Frederick Robert Carrick

University of Central Florida College of

Medicine, United States

Reviewed by:

Maria Pia Bucci,

Centre National de la Recherche

Scientifique (CNRS), France

Mandakini Sadhir,

University of Kentucky, United States

${ }^{*}$ Correspondence:

Guohui Nie

1349855331@qq.com

Yeen Huang

huangyeensz@163.com

${ }^{\dagger}$ These authors have contributed equally to this work

Specialty section:

This article was submitted to

Children and Health

a section of the journal

Frontiers in Pediatrics

Received: 14 May 2020

Accepted: 29 July 2020

Published: 15 September 2020

Citation:

Yan B, Lu X, Qiu Q, Nie G and Huang Y (2020) Association Between

Incorrect Posture and Adolescent Idiopathic Scoliosis Among Chinese

Adolescents: Findings From a Large-Scale Population-Based Study. Front. Pediatr. 8:548. doi: 10.3389/fped.2020.00548

\section{Association Between Incorrect Posture and Adolescent Idiopathic Scoliosis Among Chinese Adolescents: Findings From a Large-Scale Population-Based Study}

\author{
Bin Yan ${ }^{1,2,3}$, Xinhai Lu ${ }^{1,2,3}$, Qihua Qiu ${ }^{1,2,3}$, Guohui Nie ${ }^{1 * t}$ and Yeen Huang ${ }^{1,2,3 *+}$ \\ ${ }^{1}$ The First Affiliated Hospital of Shenzhen University, Shenzhen, China, ${ }^{2}$ Department of Spine Surgery, The Shenzhen Second \\ People's Hospital, Shenzhen, China, ${ }^{3}$ Shenzhen Youth Spine Health Center, Shenzhen, China
}

Objectives: Adolescent idiopathic scoliosis (AIS) affects between 1 and $4 \%$ of adolescents, and severe curvature may be related to their adverse long-term outcomes. However, whether the change in body appearance is related to AIS remains largely unclear. We aimed to explore the association between incorrect posture and AIS among Chinese adolescents.

Methods: Data were collected from a population-based $(595,057)$ school scoliosis screening program in China. A sample of 3,871 adolescents was classified as cases with a diagnosed radiological lateral Cobb angle $\geq 10^{\circ}$, and 3,987 control subjects with a Cobb angle $<10^{\circ}$ were randomly selected from the screening system. Adolescents were accessed with demographic information and incorrect posture measured by visual inspection of physical signs, Adam's forward bending test (FBT), and the angle of trunk rotation (ATR). Logistic regression (LR) models were used to examine the associations.

Results: Multivariate LR showed that shoulder-height difference, scapula tilt, lumbar concave, and pelvic tilt were associated with AIS. Adolescents with angle of thoracic rotation $\geq 5^{\circ}$ [adjusted odds ratio $(\mathrm{AOR})=5.33-14.67, P<0.001$ ], thoracolumbar rotation $\geq 5^{\circ}(\mathrm{AOR}=4.61-5.79, P<0.001)$, or lumbar rotation $\geq 5^{\circ}(\mathrm{AOR}=7.49-7.85$, $P<0.001$ ) were at especially higher risk for AIS than those with ATR $<5^{\circ}$.

Conclusions: Incorrect posture may be the potential risk factor for developing AIS, and $A T R \geq 5^{\circ}$ was an important indicator for predicting the occurrence of scoliosis. Early monitoring of incorrect posture for school adolescents should be considered as a routine intervention to effectively identify the progress of scoliosis.

Keywords: adolescent, scoliosis, incorrect posture, risk factor, screening 


\section{INTRODUCTION}

Adolescents idiopathic scoliosis (AIS) is the most common form of spine deformity that has a radiological lateral Cobb angle of at least $10^{\circ}(1)$. Evidence showed that AIS affects $1-4 \%$ of adolescents in the early stages of puberty, and severe spinal curvature might be associated with their adverse long-term health outcomes (e.g., pulmonary disorders, back pain, disability, psychological effects, and reduced quality of life) $(2,3)$. In China, there are more than 300 million adolescents aged $10-18$ years, and the overall prevalence of AIS was reported to be $5.14 \%$ (4), so there could be millions with AIS. Although effective treatment (brace or surgery) has been recommended for AIS in preventing further progression $(5,6)$, it is believed that early detection of irregular physical signs related to scoliosis enables effective intervention on relatively small curves.

So far, the etiology of AIS is still largely unclear. Some potential influencing factors (such as genetic basis, neurophysiological dysfunction, or skeletal growth) have been identified but not confirmed (2). In previous studies, many researchers have discussed how a patient's body asymmetry [e.g., shoulder imbalance (7), scapular tilt (8), and asymmetric spinal loading (9)] occurred after being diagnosed with scoliosis. However, in our previous large-scale scoliosis screening study (10), we found that the majority of adolescents with scoliosis could be screened for body asymmetry (e.g., shoulder-height difference, scapula prominence, lumbar concave, etc.) before being diagnosed. Therefore, we speculated that some asymmetric manifestations of body structure and function may be recognized before scoliosis occurred.

Incorrect posture refers to an abnormal body state in which the individual's body cannot maintain a standing stability and normal function of tissues and organs in an upright body posture $(10,11)$. Our previous population-based $(595,057)$ study showed that $65.3 \%$ of primary and middle school students were screened to have incorrect posture (10), and incorrect standing posture in adolescents was shown to be associated with spinal pain which may be one of the signs of progressing to scoliosis $(12,13)$. However, whether incorrect body posture is directly related to scoliosis remains largely unclear.

Therefore, we collected data from the 2019 Chinese Schoolbased Scoliosis Screening Program (CSSSP) to explore the potential association between incorrect posture and AIS. Our findings will help to comprehensively understand the potential risk factors associated with scoliosis and provide targeted intervention strategies for AIS.

\section{METHODS}

\section{Participants and Data Collection}

Data of our large-scale population-based $(595,057)$ study was collected from the 2019 CSSSP in southern China, which was an ongoing school-based scoliosis screening program targeted for Chinese children and adolescents (age, 6-18 years old) (14). CSSSP is part of the national public health project, collecting a large population-based scoliosis-related data in southern China every year since 2013, and conducted and administered by the
Shenzhen Youth Spine Health Center (SYSHC) of the Shenzhen Second People's Hospital. Detailed sampling method could be found in our previous study (10). Data were collected from February 2019 to January 2020 based on the scoliosis screening network system. Finally, a sample of 3,871 subjects were classified as the AIS group with a diagnosed radiological lateral Cobb angle $\geq 10^{\circ}$, and 3,987 randomly selected control subjects were categorized as the non-AIS group with a Cobb angle $<10^{\circ}$.

\section{School Scoliosis Screening Program}

The screening program for AIS was conducted by the SYSHC of the Shenzhen Second People's Hospital using a national scoliosis screening standardized protocol (GB/T 16133-2014) (15). School screening was performed by two independent experienced rehabilitation therapists using the visual inspection of physical signs, Adam's forward bending test (FBT), and measurement of the angle of trunk rotation (ATR). If the judgments of the two therapists were inconsistent, the third therapist would make the final decision to minimize the subjective bias. More detailed methods and processes for scoliosis screening could be found in our previous published study (10).

\section{Measures}

\section{Incorrect Posture}

Incorrect posture was assessed by visual inspection, the Adam's FBT, and the ATR. The standard visual inspection was performed in the upright position, and the examiners checked for spine alignment, shoulder asymmetry (e.g., shoulder-height difference), scapula prominence (e.g., scapula tilt), hip and pelvic obliquity (e.g., pelvic tilt), back symmetry (e.g., flat back, thoracic kyphosis), lumbar curvature (e.g., lumbar concave, lumbar kyphosis), distance of hands from the flanks, and length of the lower limbs (16). The Adam's FBT was performed with the student's feet placed together, knees straight, while bending at the hips to nearly $90^{\circ}$ with their arms freely hanging forward, palms together (17). Students with any significant physical signs were recorded. The ATR was measured with a scoliometer for quantitative assessment of the angle of thoracic rotation, angle of lumbar rotation, and angle of thoracolumbar rotation (18). Students with an ATR $\geq 5^{\circ}$ or with one or more significant physical signs were identified as having an incorrect posture. When students had an ATR $\geq 5^{\circ}$ or with two or more significant physical signs of scoliosis, they would be rescreened by specially trained physicians and referred for a standing posteroanterior radiograph of the whole spine for final diagnoses (4), and those who were found to have a Cobb angle $\geq 10^{\circ}$ measured by two independent experienced observers would be confirmed as AIS (2).

\section{Demographic Information}

Demographic characteristics included gender, age, ethnic group, and clinician's recommendations. Students' gender (boys or girls), age (years, calculated from the date of screening minus the date of birth), and ethnic group ("Han" or "minorities") (19) was obtained based on their identification (ID) card information. Clinician's recommendations were categorized as observation, exercise intervention, brace, and brace or surgery 
according to the International Society on Scoliosis Orthopedic and Rehabilitation Treatment (SOSORT) guidelines. All the above student information has been linked to the scoliosis screening network system.

\section{Statistical Analysis}

Descriptive analyses were conducted to describe the demographic characteristics and prevalence of incorrect posture among adolescents stratified by AIS, chi-square $\left(\chi^{2}\right)$ test or $t$-test was used to compare the differences between non-AIS and AIS groups. Furthermore, univariate logistic regression (LR) models were applied to preliminarily explore the association between incorrect posture and AIS, and multivariate LR were conducted to test the independent effects of each indicator of incorrect posture. Odds ratios (ORs), adjusted odds ratios (AORs), and 95\% confidence intervals (CIs) were obtained from the LR models. Missing data accounted for $<1.2 \%$ for all relevant variables and were eliminated from the statistical analysis. A two-tailed $P<0.05$ was considered statistically significant. All statistical analyses were conducted using IBM SPSS version 24.0 (IBM Corp, Armonk, NY, USA).

\section{RESULTS}

\section{Demographic Characteristics of Participants Stratified by the Occurrence of Adolescent Idiopathic Scoliosis}

As shown in Table 1, of the total sample analyzed, 3,871 (49.3\%) adolescents were diagnosed with AIS (Cobb $\left.\geq 10^{\circ}\right)$, 3,987 (50.7\%) adolescents were non-AIS ( $\left.\mathrm{Cobb}<10^{\circ}\right)$. AIS was more common in girls than in boys ( 64.8 vs. $36.1 \%, P<0.001)$, and the girls-to-boys ratio was 1.8:1 in AIS group. The mean (standard deviation) age of the AIS group was higher than that in the non-AIS group $(13.1 \pm 1.9$ vs. $12.7 \pm 2.0, P<0.001)$, the proportion of people aged 16-19 years in the AIS group was greater than that in the non-AIS group ( 13.3 vs. $8.4 \%, P<0.001$ ). For patients with AIS, 3,127 (80.8\%) patients had Cobb angles between $10^{\circ}$ and $25^{\circ}, 742(19.2 \%)$ patients were measured with Cobb angles between $26^{\circ}$ and $45^{\circ}$, and patients in these two groups were required to perform exercise intervention and wear braces, respectively. Only two patients with a Cobb angle $>45^{\circ}$ underwent surgery.

\section{Prevalence of Incorrect Posture Among Chinese Adolescents Stratified by the Occurrence of Adolescent Idiopathic Scoliosis}

As shown in Table 2, except for lumbar kyphosis, the prevalence of all other indicators of incorrect posture were different between the AIS group and the non-AIS group $(P<0.001)$. Compared to the non-AIS group, the frequencies of adolescents with angles of thoracic rotation $\geq 5^{\circ}$, thoracolumbar rotation $\geq 5^{\circ}$, and lumbar rotation $\geq 5^{\circ}$ were significantly greater in the AIS group $(P<0.001)$.
TABLE 1 | Demographic characteristics of participants stratified by the occurrence of AIS $(N=7,858)$.

\begin{tabular}{|c|c|c|c|c|}
\hline \multirow[t]{2}{*}{ Variables } & $\begin{array}{l}\text { Non-AIS group } \\
(\text { Cobb <10 })\end{array}$ & $\begin{array}{l}\text { AIS group } \\
\left(\text { Cobb } \geq 10^{\circ}\right)\end{array}$ & $\chi^{2} / t$ & $\boldsymbol{P}$ \\
\hline & $n(\%)$ & $n(\%)$ & & \\
\hline Total & 3,987 (100.0) & $3,871(100.0)$ & - & - \\
\hline Gender & & & 647.00 & $<0.001$ \\
\hline Boys & 2,548 (63.9) & 1,363 (35.2) & & \\
\hline Girls & 1,439 (36.1) & $2,508(64.8)$ & & \\
\hline Age (years) & & & 95.64 & $<0.001$ \\
\hline $7-12$ & 1,970 (49.4) & 1,537 (39.7) & & \\
\hline $13-15$ & 1,683 (42.2) & $1,819(47.0)$ & & \\
\hline $16-19$ & 334 (8.4) & 515 (13.3) & & \\
\hline Ethnic group & & & 0.05 & 0.819 \\
\hline Han & $3,871(97.1)$ & 3,755 (97.0) & & \\
\hline Minorities & $116(2.9)$ & $116(3.0)$ & & \\
\hline $\begin{array}{l}\text { Distribution of Cobb } \\
\text { angle }\end{array}$ & & & - & - \\
\hline$<10^{\circ}$ & 3,987 (100.0) & & & \\
\hline $10^{\circ}-25^{\circ}$ & - & 3,127 (80.8) & & \\
\hline $26^{\circ}-45^{\circ}$ & - & $742(19.2)$ & & \\
\hline$>45^{\circ}$ & - & $2(<0.001)$ & & \\
\hline $\begin{array}{l}\text { Clinician's } \\
\text { recommendations }\end{array}$ & & & - & - \\
\hline Observation & 3,987 (100.0) & - & & \\
\hline Exercise intervention & - & 3,127 (80.8) & & \\
\hline Brace & - & $742(19.2)$ & & \\
\hline Brace or surgery & - & $2(<0.001)$ & & \\
\hline
\end{tabular}

AIS, adolescent idiopathic scoliosis; $n$, number; $S D$, standard deviation.

\section{Association Between Incorrect Posture and Adolescent Idiopathic Scoliosis Among Chinese Adolescents}

As shown in Table 3, in the univariate LR model (Model 1), gender, age, shoulder-height difference, scapula tilt, lumbar concave, pelvic tilt, thoracic kyphosis, angle of thoracic rotation, angle of thoracolumbar rotation, and angle of lumbar rotation were significantly associated with AIS $(P<0.001)$. In the multivariate LR method (Model 2), except for thoracic kyphosis, the associations of the above indicators with AIS were weakened, but there was still statistical difference $(P<0.01)$. Adolescents with angles of thoracic rotation $\geq 5^{\circ}$, thoracolumbar rotation $\geq 5^{\circ}$, and lumbar rotation $\geq 5^{\circ}$ were significantly associated with a higher risk for developing AIS than those with ATR $<5^{\circ}$ $(P<0.001)$.

\section{DISCUSSION}

In our 2019 CSSSP, adolescents participating in the school screening would undergo a rigorous physical examination by two independent experienced therapists, and scoliosis patients could be effectively identified by some signs of incorrect posture assessed by the visual inspection of physical signs, the Adam's FBT, and measurement of the ATR. Using a largescale population-based $(595,057)$ dataset, we further clarified the role of incorrect posture in identifying AIS and non-AIS 
TABLE 2 | Prevalence of incorrect posture among Chinese adolescents stratified by the occurrence of AIS $(N=7,858)$.

\begin{tabular}{cccc}
\hline Variables & $\begin{array}{c}\text { Non-AIS group } \\
\left(\text { Cobb }<10^{\circ}\right)\end{array}$ & $\begin{array}{c}\text { AIS group } \\
\left(\text { Cobb } \geq 10^{\circ}\right)\end{array}$ & \\
\cline { 1 - 3 } & $n(\%)$ & $n(\%)$ \\
\hline
\end{tabular}

\section{Total}

$3,987(100.0) \quad 3,871(100.0)$

Incorrect posture ${ }^{a}$

\begin{tabular}{|c|c|c|c|c|}
\hline Shoulder-height difference & & & $1,475.79$ & $<0.001$ \\
\hline Normal & $3,469(87.0)$ & $1,796(46.4)$ & & \\
\hline Left shoulder height & $327(8.2)$ & $1103(28.5)$ & & \\
\hline Right shoulder height & $191(4.8)$ & $972(25.1)$ & & \\
\hline Scapula tilt & & & $2,460.31$ & $<0.001$ \\
\hline Normal & $3,405(85.4)$ & $1,173(30.3)$ & & \\
\hline Tilt to the left & $379(9.5)$ & $1,529(39.5)$ & & \\
\hline Tilt to the right & $203(5.1)$ & $1,169(30.2)$ & & \\
\hline Lumbar concave & & & $1,533.17$ & $<0.001$ \\
\hline Normal & $3,624(90.9)$ & $1,970(50.9)$ & & \\
\hline Left concave & $140(3.5)$ & $794(20.5)$ & & \\
\hline Right concave & $223(5.6)$ & $1,107(28.6)$ & & \\
\hline Pelvic tilt & & & 582.34 & $<0.001$ \\
\hline Normal & $3,832(96.1)$ & $3,039(78.5)$ & & \\
\hline Tilt to the left & $104(2.6)$ & $298(7.7)$ & & \\
\hline Tilt to the right & $51(1.3)$ & $534(13.8)$ & & \\
\hline Flat back & & & 21.51 & $<0.001$ \\
\hline Normal & 3,979 (99.8) & $3,832(99.0)$ & & \\
\hline Abnormal & $8(0.2)$ & $39(1.0)$ & & \\
\hline Thoracic kyphosis & & & 62.52 & $<0.001$ \\
\hline Normal & 3,947 (99.0) & 3,728 (96.3) & & \\
\hline Abnormal & $40(1.0)$ & $143(3.7)$ & & \\
\hline Lumbar kyphosis & & & 2.44 & 0.118 \\
\hline Normal & $3,971(99.6)$ & 3,863 (99.8) & & \\
\hline Abnormal & $16(0.4)$ & $8(0.2)$ & & \\
\hline Angle of thoracic rotation & & & $1,209.99$ & $<0.001$ \\
\hline Normal (ATR: $0^{\circ}-4^{\circ}$ ) & $3,800(95.3)$ & $2,474(63.9)$ & & \\
\hline Rotate to the left $\left(A T R \geq 5^{\circ}\right)$ & $80(2.0)$ & $395(10.2)$ & & \\
\hline Rotate to the right $\left(A T R \geq 5^{\circ}\right)$ & $107(2.7)$ & $1,002(25.9)$ & & \\
\hline $\begin{array}{l}\text { Angle of thoracolumbar } \\
\text { rotation }\end{array}$ & & & 185.08 & $<0.001$ \\
\hline Normal (ATR: $\left.0^{\circ}-4^{\circ}\right)$ & $3,883(97.4)$ & $3,484(90.0)$ & & \\
\hline Rotate to the left $\left(\right.$ ATR $\geq 5^{\circ}$ ) & $40(1.0)$ & $186(4.8)$ & & \\
\hline Rotate to the right $\left(A T R \geq 5^{\circ}\right.$ ) & $64(1.6)$ & $201(5.2)$ & & \\
\hline Angle of lumbar rotation & & & $1,161.88$ & $<0.001$ \\
\hline Normal (ATR: $0^{\circ}-4^{\circ}$ ) & 3,636 (91.2) & $2,237(57.8)$ & & \\
\hline Rotate to the left $\left(\right.$ ATR $\left.\geq 5^{\circ}\right)$ & $271(6.8)$ & $1,212(31.3)$ & & \\
\hline Rotate to the right $\left(A T R \geq 5^{\circ}\right.$ ) & $80(2.0)$ & $422(10.9)$ & & \\
\hline
\end{tabular}

AIS, adolescent idiopathic scoliosis; $n$, number; ATR, angle of trunk rotation.

aIncorrect posture was defined as a participant who was screened out one or more to the following physical signs: shoulder-height difference, scapula tilt, lumbar concave, pelvic tilt, flat back, thoracic kyphosis, lumbar kyphosis, angle of thoracic rotation $\geq 5^{\circ}$, angle of thoracolumbar rotation $\geq 5^{\circ}$, angle of lumbar rotation $\geq 5^{\circ}$.

groups and found that ATR $\geq 5^{\circ}$ might have the strongest associations with the occurrence of scoliosis in adolescents. This indicated that incorrect posture could be a critical indicator for school scoliosis screening, especially the indicator of ATR. Our findings could help resolve the problem of low positive predictive value (PPV) and high referral rate in school-based screening for scoliosis.
TABLE 3 | Association between incorrect posture and AIS among Chinese adolescents $(N=7,858)$.

\begin{tabular}{|c|c|c|c|c|c|c|}
\hline \multirow[t]{2}{*}{ Variables } & \multicolumn{3}{|c|}{ 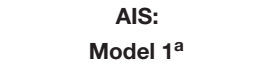 } & \multicolumn{3}{|c|}{$\begin{array}{c}\text { AIS: } \\
\text { Model } 2^{b}\end{array}$} \\
\hline & OR & $95 \% \mathrm{Cl}$ & $P$ & AOR & $95 \% \mathrm{Cl}$ & $P$ \\
\hline \multicolumn{7}{|l|}{ Gender } \\
\hline Boys & 1.00 & & & 1.00 & & \\
\hline Girls & 3.26 & 2.69-3.96 & $<0.001$ & 1.99 & $1.42-2.78$ & $<0.001$ \\
\hline Age (years) & 1.14 & $1.08-1.19$ & $<0.001$ & 1.09 & $1.02-1.17$ & $<0.001$ \\
\hline $7-12$ & 1.00 & & & 1.00 & & \\
\hline $13-15$ & 1.38 & $1.09-1.75$ & 0.007 & 1.18 & $0.83-1.69$ & 0.354 \\
\hline $16-19$ & 1.99 & $1.35-2.93$ & $<0.001$ & 1.96 & $1.12-3.42$ & 0.018 \\
\hline \multicolumn{7}{|l|}{ Ethnic group } \\
\hline Han & 1.00 & & & 1.00 & & \\
\hline Minorities & 0.98 & $0.82-1.13$ & 0.889 & 0.99 & $0.86-1.12$ & 0.631 \\
\hline
\end{tabular}

Incorrect posture

Shoulder-height difference

Normal

Left shoulder height

Right shoulder height

Scapula tilt

Normal

Tilt to the left

Tilt to the right

Lumbar concave

Normal

Left concave

Right concave

Pelvic tilt

Normal

Tilt to the left

Tilt to the right

Flat back

Normal

Abnormal

Thoracic kyphosis

Normal

Abnormal

Lumbar kyphosis

Normal

Abnormal

Angle of thoracic rotation

Normal (ATR: $0^{\circ}-4^{\circ}$ )

Rotate to the left $\left(A T R \geq 5^{\circ}\right)$

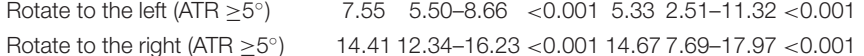

Angle of thoracolumbar rotation

Normal (ATR: $\left.0^{\circ}-4^{\circ}\right)$

Rotate to the left $\left(A T R \geq 5^{\circ}\right)$

Rotate to the right $\left(A T R \geq 5^{\circ}\right)$

Angle of lumbar Rotation

Normal (ATR: $0^{\circ}-4^{\circ}$ )

$\begin{array}{lll}1.00 & 1.00\end{array}$

$\begin{array}{lllll}6.56 & 4.92-8.74<0.001 & 2.63 & 1.53-4.53<0.001\end{array}$

$9.817 .93-11.89<0.0013 .72 \quad 1.99-6.97<0.001$

$1.00 \quad 1.00$

$11.719 .89-12.41<0.0012 .34 \quad 1.38-3.97<0.001$

$16.5513 .75-18.30<0.001 \quad 2.89 \quad 1.57-5.30 \quad 0.001$

$1.00 \quad 1.00$

$10.568 .09-12.72<0.0013 .62 \quad 1.88-6.96<0.001$

$9.157 .62-11.66<0.0013 .81 \quad 2.30-6.28<0.001$

$1.00 \quad 1.00$

$\begin{array}{llllll}3.66 & 2.26-5.94 & <0.001 & 0.35 & 0.17-0.74 & 0.006\end{array}$

$12.608 .91-14.99<0.001 \quad 1.42 \quad 0.60-3.35 \quad 0.420$

$1.00 \quad 1.00$

$2.59 \quad 0.92-3.32 \quad 0.852 \quad 1.28 \quad 0.76-3.19 \quad 0.445$

$1.00 \quad 1.00$

$\begin{array}{llllll}3.82 & 2.82-5.03 & <0.001 & 1.98 & 0.83-4.37 & 0.117\end{array}$

$\begin{array}{lll}1.00 & 1.00\end{array}$

$\begin{array}{llllll}0.51 & 0.09-2.77 & 0.431 & 0.10 & 0.01-1.77 & 0.990\end{array}$

$1.00 \quad 1.00$

$1.00 \quad 1.00$

$8.11 \quad 6.47-9.57<0.0015 .792 .30-14.58<0.001$

$5.60 \quad 3.96-7.60<0.0014 .612 .06-10.31<0.001$

$1.00 \quad 1.00$

$7.25 \quad 5.38-9.77<0.0017 .494 .79-11.71<0.001$

$8.526 .09-10.26<0.0017 .853 .68-16.73<0.001$

Rotate to the left (ATR $\left.\geq 5^{\circ}\right)$

Rotate to the right $\left(A T R \geq 5^{\circ}\right)$

AIS, adolescent idiopathic scoliosis; OR, odds ratio; AOR, adjusted odds ratio; Cl, confidence interval; ATR, angle of trunk rotation.

a Univariate logistic regression model.

${ }^{b}$ Multivariate logistic regression model.

Using incorrect posture as the indicators for screening scoliosis, we found that the prevalence of shoulder-height difference, scapula tilt, lumbar concave, pelvic tilt, flat back, 
and thoracic kyphosis was significantly higher in adolescents diagnosed with AIS than those with non-AIS, which were consistent with the previous research (20). Furthermore, multivariate LR model showed that shoulder-height difference, scapula tilt, and lumbar concave were independently associated with AIS. Using biomechanics and three-dimensional spatial positioning methods, some researchers speculated the alterations of shoulder, scapula, and lumbar spine could be considered as adaptive compensation or muscle activation strategies in AIS patients (21-23). Interestingly, we found that a pelvic tilt to the left $(\mathrm{AOR}=0.35,95 \% \mathrm{CI}: 0.17-0.74)$ seems to be a protective factor for scoliosis. This may be related to the fact that most scoliosis occurs on the right side; in order to maintain the balance of the body in a sagittal standing posture, the pelvis of the individual is prone to tilt to the left under the effect of adaptive compensation (24).

The validity and reliability of using the scoliometer to measure ATR for scoliosis screening have been verified $(25,26)$, but few studies have explored the magnitude of the association between ATR and scoliosis. We filled the gaps in this field and found that the angles of thoracic rotation $\geq 5^{\circ}$, thoracolumbar rotation $\geq 5^{\circ}$, and lumbar rotation $\geq 5^{\circ}$ were significantly associated with a higher risk for AIS than those with ATR $<5^{\circ}$, in which adolescents whose angle of thoracic rotation to the right $\geq 5^{\circ}$ were 10 times more likely to develop scoliosis than adolescents with ATR $<5^{\circ}$. Studies from Pratt et al. (27) and Scutt et al. (28) both showed that a larger ATR was related to more severe scoliosis. A recent cross-sectional study indicated that rotation of the thoracic and thoracolumbar spine appeared to be the most differentiating in the diagnosis of AIS, and mild scoliosis could be found even in adolescents with a lower degree of trunk rotation $\left(4^{\circ}-6^{\circ}\right)(29)$. Our research supported the results of previous studies and further quantified the relationship between ATR and AIS, which could provide an objective reference for more accurate identification of scoliosis patients in large-scale school screening.

The benefits and harms of school screening for AIS remain controversial. This is mainly related to the relatively low PPV for identifying patients and that leads to over-referral of adolescents who do not require follow-up or radiography (3). In order to solve this problem, we put forward a new concept of "incorrect posture," which includes 10 indicators related to changes in body appearance that can be measured by visual inspection of physical signs, the Adam's FBT, and the ATR. Using this screening method, our previous findings showed a PPV of $83.8 \%$ for identifying AIS patients with a Cobb angle $\geq 10^{\circ}$ in a large-scale (961,169 adolescents) school scoliosis screening (14), which was higher than the results of the largest cohort study conducted in Hong Kong (16). This showed the feasibility and effectiveness of using incorrect posture as the identification indicator for school scoliosis screening in China.

The present study had several limitations that were worth noting. First, due to the cross-sectional design of the study, it was difficult to make causal inferences, so the possibility of reverse association might exist. Second, although each indicator of incorrect posture was judged by two independent therapists, it was still difficult to completely avoid subjective measurement bias. Third, due to the large number of adolescents needed to be screened, other influencing factors (e.g., genetics, hormone, or nutritional status) related to AIS had not been fully investigated, which might overestimate the magnitude of the association between incorrect posture and AIS. Fourth, since the nature of the cross-sectional data, we could only obtain limited information about the condition of AIS patients, the longitudinal relationship between incorrect posture and the development of AIS could not be discussed. Future prospective cohort studies would help fill the gaps in this research area. Fifth, our data mainly came from subjective physical examinations; although the measurement results in the study were assessed by two independent observers, potential measurement bias between observers for the severity of incorrect posture might exist.

\section{CONCLUSION}

Using a population-based screening dataset, we found that incorrect posture was associated with AIS among Chinese adolescents, and ATR $\geq 5^{\circ}$ could be considered as the best indicator to identify the occurrence of scoliosis. Early monitoring and identification of bad postures could be considered as a feasible and effective targeted intervention for preventing future AIS. More longitudinal studies to clarify the causal relationship between incorrect posture and scoliosis are needed.

\section{DATA AVAILABILITY STATEMENT}

The datasets supporting the conclusions of this article will be made available by the authors upon reasonable request.

\section{ETHICS STATEMENT}

This study was conducted in accordance with the Declaration of Helsinki and was approved by the Shenzhen Municipal Health Commission Institutional Review Board. Written informed consent was obtained from the individual(s), and minor(s)' legal guardian/next of kin, for the publication of any potentially identifiable images or data included in this article.

\section{AUTHOR CONTRIBUTIONS}

YH and GN designed and supervised the study. BY and XL collected the screening data. XL and QQ carried out the statistical analysis. BY wrote the original draft. YH and GN reviewed and corrected the revised manuscript. All authors contributed to the article and approved the submitted version.

\section{FUNDING}

This work was supported by the Scoliosis screening program for primary and secondary school students in Shenzhen (Project number: SFG [2019] No. 780), study on the training effects of adolescent idiopathic scoliosis in Shenzhen (Project number: No. 20193357005), and Shenzhen key medical discipline construction fund. 


\section{ACKNOWLEDGMENTS}

The authors would like to express sincere respect to the local health professional and education bureau for their valuable contribution in setting up the school screening program.

\section{REFERENCES}

1. Altaf F, Gibson A, Dannawi Z, Noordeen H. Adolescent idiopathic scoliosis. BMJ. (2013) 346:f2508. doi: 10.1136/bmj.f2508

2. Cheng JC, Castelein RM, Chu WC, Danielsson AJ, Dobbs MB, Grivas TB, et al. Adolescent idiopathic scoliosis. Nat Rev Dis Primers. (2015) 1:15030. doi: 10.1038/nrdp.2015.30

3. Grossman DC, Curry SJ, Owens DK, Barry MJ, Davidson KW, Doubeni CA, et al. Screening for adolescent idiopathic scoliosis: US preventive services task force recommendation statement. JAMA. (2018) 319:16572. doi: 10.1001/jama.2017.19342

4. Hengwei F, Zifang H, Qifei W, Weiqing T, Nali D, Ping Y, et al. Prevalence of idiopathic scoliosis in Chinese schoolchildren: a large, population-based study. Spine. (2016) 41:259-64. doi: 10.1097/BRS.0000000000001197

5. Zheng Y, Dang YN, Yang Y, Li HB, Zhang LJ, Lou EHM, et al. Whether orthotic management and exercise are equally effective to the patients with adolescent idiopathic scoliosis in mainland China? A randomized controlled trial study. Spine. (2018) 43:E494-503. doi: 10.1097/BRS.0000000000002412

6. Toru M, Yosuke K, Makoto M, Nakao Y. Effectiveness of brace treatment for adolescent idiopathic scoliosis. Scoliosis. (2015) 10(Suppl 2):S12. doi: 10.1186/1748-7161-10-S2-S12

7. Zhang SF, Zhang L, Feng XM, Yang HL. Incidence and risk factors for postoperative shoulder imbalance in scoliosis: a systematic review and metaanalysis. Eur Spine J. (2018) 27:358-69. doi: 10.1007/s00586-017-5289-y

8. Lin JJ, Chen WH, Chen PQ, Tsauo JY. Alteration in shoulder kinematics and associated muscle activity in people with idiopathic scoliosis. Spine. (2010) 35:1151-7. doi: 10.1097/BRS.0b013e3181cd5923

9. Villemure I, Aubin CE, Dansereau J, Labelle H. Simulation of progressive deformities in adolescent idiopathic scoliosis using a biomechanical model integrating vertebral growth modulation. J Biomech Eng. (2002) 124:78490. doi: 10.1115/1.1516198

10. Yang L, Lu XH, Yan B, Huang YE. Prevalence of incorrect posture among children and adolescents finding from a large population-based study in China. Science. (2020) 23:101043. doi: 10.1016/j.isci.2020.101043

11. Dolphens M, Cagnie B, Coorevits P, Vanderstraeten G, Cardon G, D'hooge R, et al. Sagittal standing posture and its association with spinal pain: a school-based epidemiological study of 1196 Flemish adolescents before age at peak height velocity. Spine. (2012) 37:165766. doi: 10.1097/BRS.0b013e3182408053

12. Nault ML, Allard P, Hinse S, Blanc RL, Caron O, Labelle H, et al. Relations between standing stability and body posture parameters in adolescent idiopathic scoliosis. Spine. (2002) 27:1911-7. doi: 10.1097/00007632-200209010-00018

13. Nissinen M, Heliövaara M, Seitsamo J, Poussa M. Trunk asymmetry, posture, growth, and risk of scoliosis. A three-year followup of Finnish prepubertal school children. Spine. (1993) 18:8-13. doi: 10.1097/00007632-199301000-00002

14. Yan B, Lu XH, Nie GH, Huang YE. China urgently needs a nationwide scoliosis screening system. Acta Paediatr. (2020). doi: 10.1111/apa.15326. [Epub ahead of print].

15. National Health and Family Planning Commission of the people's Republic of China and National Standardization Administration Commission of China. Screening of spinal curvature abnormality of children and adolescents (GB/T 16133-2014). Available online at: http://ddedu.dandong.gov.cn/docs/201906/20190618095730294569.pdf (accessed on 14 May 2020).

16. Luk KD, Lee CF, Cheung KMC, Cheng JCY, Ng BKW, Lam TP, et al. Clinical effectiveness of school screening for adolescent idiopathic scoliosis: a large population-based retrospective cohort study. Spine. (2010) 35:160714. doi: 10.1097/BRS.0b013e3181c7cb8c
Moreover, we would like to express our heartfelt thanks to all the rehabilitation therapists for their help with the scoliosis screening and data collection. Finally, we are very grateful for the professional language guidance provided by Ms. Qiaohong Chen.
17. Bunnell WP. An objective criterion for scoliosis screening. J Bone Joint Surg Am. (1984) 66:1381-7. doi: 10.1007/BF00269920

18. Cote P, Kreitz BG, Cassidy JD, Dzus AK, Martel J. A study of the diagnostic accuracy and reliability of the Scoliometer and Adam's forward bend test. Spine. (1998) 23:796-803. doi: 10.1097/00007632-19980401000011

19. Xu L, Qin X, Sun W, Qiao J, Qiu Y, Zhu Z, et al. Replication of association between 53 single-nucleotide polymorphisms in a DNA-based diagnostic test and AIS progression in Chinese Han population. Spine. (2016) 41:30610. doi: 10.1097/BRS.0000000000001203

20. Raso VJ, Lou E, Hill DL, Mahood JK, Moreau MJ, Durdle NG. Trunk distortion in adolescent idiopathic scoliosis. J Pediatr Orthop. (1998) 18:2226. doi: 10.1097/01241398-199803000-00017

21. Kwan MK, Wong KA, Lee CK, Chan CYW. Is neck tilt and shoulder imbalance the same phenomenon? A prospective analysis of 89 adolescent idiopathic scoliosis patients (Lenke type 1 and 2). Eur Spine J. (2016) 25:4018. doi: 10.1007/s00586-015-4016-9

22. Turgut E, Gur G, Ayhan C,Yakut Y, Baltaci G. Scapular kinematics in adolescent idiopathic scoliosis: a three-dimensional motion analysis during multiplanar humeral elevation. J Biomech. (2017) 61:224-31. doi: 10.1016/j.jbiomech.2017.07.029

23. Stokes IAF, Gardner-Morse M. Muscle activation strategies and symmetry of spinal loading in the lumbar spine with scoliosis. Spine. (2004) 29:21037. doi: 10.1097/01.brs.0000141182.42544.1f

24. Saetang L, Sakulsriprasert P, Vongsirinavarat M. The adaptive patterns of pelvic alignment in individuals with adolescent idiopathic scoliosis. J Med Assoc Thai. (2015) 98(Suppl 5):S119-24.

25. Korovessis PG. Scoliometer is useful instrument with high reliability and repeatability. Spine. (1999) 24:3078. doi: 10.1097/00007632-199902010-00027

26. Amendt LE, Ause-Ellias KL, Eybers JL, Wadsworth CT, Nielsen $\mathrm{DH}$, Weinstein SL. Validity and reliability testing of the scoliometer. Phys Ther. (1990) 70:108-17. doi: 10.1093/ptj/ 70.2.108

27. Pratt RK, Burwell RG, Cole AA, Webb JK. Patient and parenta perception of adolescent idiopathic scoliosis before and after surgery in comparison with surface and radiographic measurements. Spine. (2002) 27:1543-52. doi: 10.1097/00007632-20020715000012

28. Scutt ND, Dangerfield PH, Dorgan JC. The relationship between surface and radiological deformity in adolescent idiopathic scoliosis: effect of change in body position. Eur Spine J. (1996) 5:85-90. doi: 10.1007/BF00298386

29. Adamczewska K, Wiernicka M, Malchrowicz-Moko E, Malchrowicz-Mośko E, Małecka J, Lewandowski J. The angle of trunk rotation in school children: a study from an idiopathic scoliosis screening. Prevalence and optimal age screening value. Int J Environ Res Public Health. (2019) 16:3426. doi: 10.3390/ijerph16183426

Conflict of Interest: The authors declare that the research was conducted in the absence of any commercial or financial relationships that could be construed as a potential conflict of interest.

Copyright (C) 2020 Yan, Lu, Qiu, Nie and Huang. This is an open-access article distributed under the terms of the Creative Commons Attribution License (CC BY) The use, distribution or reproduction in other forums is permitted, provided the original author(s) and the copyright owner(s) are credited and that the original publication in this journal is cited, in accordance with accepted academic practice. No use, distribution or reproduction is permitted which does not comply with these terms. 